\title{
Dapsone induced hypersensitivity syndrome: a case report
}

\author{
Sarika Kolli*, Priyanka Suhagiya
}

Department of Pharm. D, CMR College of Pharmacy, Medchal, Hyderabad, Telangana, India

Received: 24 July 2020

Accepted: 02 September 2020

*Correspondence:

Sarika Kolli,

Email: sarikakolli95@gmail.com

Copyright: (c) the author(s), publisher and licensee Medip Academy. This is an open-access article distributed under the terms of the Creative Commons Attribution Non-Commercial License, which permits unrestricted non-commercial use, distribution, and reproduction in any medium, provided the original work is properly cited.

\begin{abstract}
Dapsone is an anti-leprotic drug. It may cause a severe adverse effect known as dapsone hypersensitivity syndrome which involves fever, chills, rash, and internal organ involvement. We report a case of a patient who is 30 years old male presented with fever, skin desquamation, and hepatitis. The case is being reported to emphasize the need to diagnose and manage dapsone hypersensitivity syndrome.
\end{abstract}

Keywords: Dapsone, Hypersensitivity syndrome, Hepatitis

\section{INTRODUCTION}

Dapsone helps in treating infections, immunological, and hypersensitivity disorders. Dapsone adverse effects are mainly dose unrelated skin hypersensitivity reactions and dose-related haemolytic anaemia and methemoglobinemia. ${ }^{1}$ Drug-induced hypersensitivity syndrome is characterized by fever, rash, dehydration, and internal organ involvement. Abnormal liver function tests, low haemoglobin, and high bilirubin levels were also found. ${ }^{2}$

\section{CASE REPORT}

A 30 year old male patient was brought with fever since 1 month associated with chills and rigors, cough with whitish sputum, grade 1 shortness of breath, burning type of chest pain, desquamation of skin, body pains and jaundice. One month before current admission, he was started on multidrug therapy (rifampin $600 \mathrm{mg}$, clofazimine $300 \mathrm{mg}$, and dapsone $100 \mathrm{mg} /$ day) by a dermatologist for leptospirosis. His vital signs were as follows: blood pressure- $110 / 70 \mathrm{~mm} \mathrm{Hg}$; pulse rate$126 /$ min. On physical examination, the patient was icteric positive. In the cutaneous examination, the patient had desquamation all over the body. Laboratory evaluation revealed sodium $(\mathrm{Na})-134 \mathrm{mEq} / \mathrm{l}$; potassium $(\mathrm{K})-3.3$
$\mathrm{mEq} / \mathrm{l}$; chlorine $(\mathrm{Cl})-99 \mathrm{mEq} / \mathrm{l}$; serum creatinine- 0.62 $\mathrm{mg} / \mathrm{dl}$; blood urea- $14.55 \mathrm{mg} / \mathrm{dl}$; protein- $4.25 \mathrm{~g} / \mathrm{dl}$; albumin- $2.38 \mathrm{~g} / \mathrm{dl}$; alkaline phosphatase (ALP)- $75.2 \mathrm{IU} / \mathrm{l}$ and; total serum bilirubin (TSB)- $2.38 \mathrm{mg} / \mathrm{dl}$.

His liver function tests were abnormal with decreased levels of protein and albumin and increased levels of total serum bilirubin.

Abdominal ultrasound showed coarse hepatic echotexture and bilateral mild ascites.

The patient was on multidrug therapy. Multidrug therapy was withdrawn on the day of admission and prescribed with injection ceftriaxone $1 \mathrm{~g}$, tablet doxycycline $100 \mathrm{mg}$, tablet ursodeoxycholic acid $100 \mathrm{mg}$.

A diagnosis of dapsone induced hypersensitivity syndrome was made based on the history of dapsone intake, followed by fever, skin desquamation, and hepatitis. He was started on hydrocortisone $100 \mathrm{mg}$. He improved over 5 days with gradual subsidence of fever, skin desquamation, and hepatitis. Antibiotics were discontinued after 10 days and he was discharged on day 11 of admission. On the day of discharge, the patient was advised to continue multibacillary multidrug therapy except dapsone. 


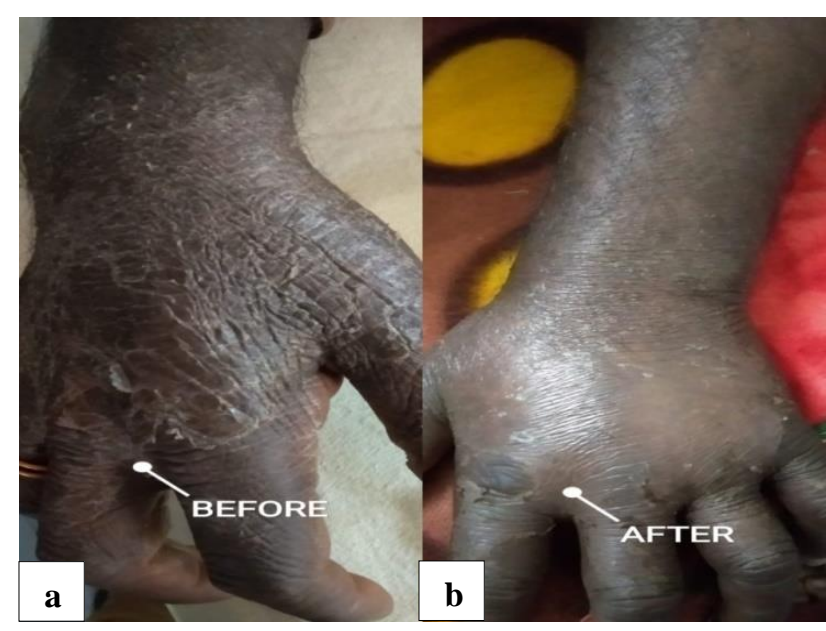

Figure 1: Clinical finding of desquamation (skin peeling) on hands in a patient with drug-induced hypersensitivity syndrome (a) before giving treatment, and (b) after giving treatment.

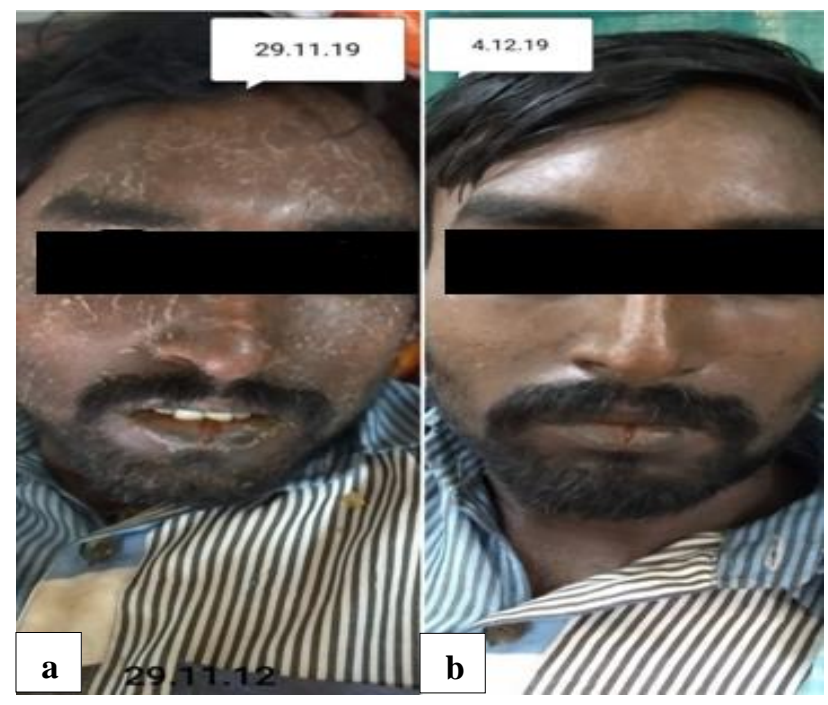

Figure 1: Clinical finding of desquamation (skin peeling) on face in a patient with drug-induced hypersensitivity syndrome (a) before giving treatment, and (b) after giving treatment.

\section{DISCUSSION}

Dapsone is used for treatment or prophylaxis of several infections, in dermatological conditions and immune thrombocytopenic purpura. ${ }^{1}$ After initiation of treatment with dapsone, drug-induced hypersensitivity syndrome (DIHS) can develop more commonly between the fourth and sixth weeks. ${ }^{2}$

Hepatitis with coarse hepatic echotexture and cutaneous manifestation like desquamation of skin are severe systemic manifestations observed in our patient. The diagnosis of DIHS is based on clinical findings of fever, desquamation of skin, and abnormal liver tests along with a history of exposure to dapsone. ${ }^{1}$
If at least two of the following signs or symptoms are present then the reaction is classified as dapsone syndrome: fever, skin eruption, lymphadenopathy, and liver pathology (hepatomegaly, jaundice, and/or abnormal liver function tests. ${ }^{3}$ Our patient had a fever, skin eruption, and abnormal liver function tests.

Differences in dapsone metabolism, which affect the production and detoxification of its reactive metabolites, might be responsible for differential susceptibility of people to the adverse effects of dapsone. ${ }^{1}$ The pathophysiological mechanism involves immune humoral response, a delayed hypersensitivity reaction, and altered hepatic metabolism including acetylation and hydroxylation with secondary toxic metabolite production. $^{2}$

Hypoalbuminemia and hyperbilirubinemia are present in our patient which are features of dapsone hypersensitivity. Hypoalbuminemia is due to the binding of dapsone to circulating serum albumin. Hyperbilirubinemia is due to hemolysis in addition to hepatotoxicity. ${ }^{4}$

DIHS can be treated by immediate withdrawal of dapsone therapy followed by supportive care of symptoms and starting treatment with oral corticosteroids.4 Corticosteroids are used for treating symptoms and prevention of progression. For mild cases, systemic corticosteroids dosed at 0.5 to $1 \mathrm{mg} /$ day and tapered over 6-8 weeks are recommended. ${ }^{5}$ Systemic corticosteroids should be initiated at a dose of 40-60 mg prednisone equivalent daily, followed by a gradual dose reduction of prednisone given over 10 weeks to prevent the rapid reconstitution of valid immune responses against various pathogens. ${ }^{6}$ Doses of corticosteroid are tapered and treatment is continued for many weeks as chances for relapses is high. ${ }^{7}$ Patients treated with systemic corticosteroids lived longer than those not treated with corticosteroids. Corticosteroids, especially if administered in the acute stage, may improve the long term outcome. ${ }^{6}$

Drugs more often reported to cause drug hypersensitivity syndrome are dapsone, sulphonamides, trimethoprim. ${ }^{7}$

Antibiotic therapy is given to the patient based on clinical symptoms, including positive skin cultures, a sudden drop in temperature, or deterioration of the patient's medical condition. $^{5}$

For paucibacillary leprosy, give monthly rifampicin pulse and daily clofazimine therapy. For multibacillary leprosy, give monthly rifampicin and clofazimine pulse and daily clofazimine therapy. ${ }^{4}$

\section{CONCLUSION}

Prompt and timely diagnosis along with identification and cessation of suspected drug and appropriate management is recommended for physicians. 
In the case of patients presenting with dermatological disorders, medications including start dates are reviewed along with the patient's subjective and past medical and medication history.

Funding: No funding sources

Conflict of interest: None declared

Ethical approval: Not required

\section{REFERENCES}

1. Vinod KV, Arun K, Dutta TK. Dapsone hypersensitivity syndrome: A rare life

2. threatening complication of dapsone therapy. J Pharmacol Pharmacother. 2013;4(2):158-60.

3. Gavilanes MC, Palacio AL, Chellini PR, Nery JA, Rego JG. Dapsone hypersensitivity syndrome in a lepromatous leprosy patient- A case report. Lepr Rev. 2015;86:186-90.

4. Alungal J, Abdulla MC, Kunnummal NK, Sivadasan A. Dapsone- induced hypersensitivity syndrome, haemolytic anemia, and severe agranulocytosis. Int $\mathbf{J}$ Nutr Pharmacol Neurol Dis. 2015;5:113-6.

5. Grace M. An unusual case of dapsone syndrome. Indian Dermatol Online J. 2011;2:88-90.

6. Hamm RL. Drug-hypersensitivity syndrome: diagnosis and treatment. J Am Coll Clin Wound Spec. 2012;3(4):7781.

7. Watanabe H. Recent Advances in Drug-Induced Hypersensitivity Syndrome/DrugReaction with Eosinophilia and Systemic Symptoms. J Immunol Res. 2018;5163129.

8. Rademaker M, Maling T. Drug Hypersensivity syndrome. Medsafe. 2003;24(2):22-3.

Cite this article as: Kolli S, Suhagiya P. Dapsone induced hypersensitivity syndrome: a case report. Int J Basic Clin Pharmacol 2020;9:1609-11. 